\title{
Comparing Wireless LAN Standards and a Model Proposal for University Wireless Network Security with $802.1 \mathrm{x}$
}

\author{
Mehmet Kösem ${ }^{1}$, Ali Güneş ${ }^{2}$ \\ Fatih Sultan Mehmet University, İstanbul ${ }^{1}$ \\ İstanbul Aydın University, İstanbul ${ }^{2}$
}

\begin{abstract}
With the increasing use of wireless devices, information sharing, use of systems and many different applications were taken into account over Wireless Network. One of the biggest problems of Wireless network is that it might lead to many security gaps. When Universities, naturally considering academic staff, managers and student population the number of users in Wireless Network are many, therefore Universities are firms that need to be careful about security due to the different applications. In this research, the Security Methods and Standards were generally discussed, after comparing them, secure wireless network architecture has been designed for Universities and the design has been applied in Fatih Sultan Mehmet University.
\end{abstract}

Keywords: wireless networks, network security, 802.1x, campus network architecture.

\section{WIRELESS LAN STANDARDS}

With the development of technology day by day, and easy there may be many people who can follow this broadcast use of technology, improvements of Wireless LAN and Users from different authorization levels who are Standards and especially users' choice on mobile devices included in that broadcast can reach the data within the increased the interest in wireless networks. With the network. Such situations may result in security increasing use of wireless devices, information sharing, vulnerabilities in many networks. There are many use of systems and many different applications were taken standards in wireless networks. These standards can be into account over Wireless Network. But wireless classified according to their security structures that are networks are the systems of realization of communication used in local networks and wireless networks. The between air broadcasting devices and the devices that get characteristics of local wireless network standard are given that broadcast[1], during the exchange of data,

in Table-1.

Table-1Comparison of Wireless LAN Standards

\begin{tabular}{|l|l|l|l|l|l|}
\hline Standard & Release Date & Frequency Band & Data SPEED & Distance (Indoor) & Distance(Outdoor) \\
\hline 802.11 & 1997 & $2.4 \mathrm{GHz}$ & $1-2 \mathrm{Mpbs}$ & $10 \mathrm{M}$ & $75 \mathrm{M}$ \\
\hline $802.11 \mathrm{a}$ & 1999 & $5 \mathrm{Ghz}$ & $54 \mathrm{Mpbs}$ & $13 \mathrm{M}$ & $100 \mathrm{M}$ \\
\hline $802.11 \mathrm{~b}$ & 1999 & $2.4 \mathrm{GHz}$ & $11 \mathrm{Mpbs}$ & $35 \mathrm{M}$ & $110 \mathrm{M}$ \\
\hline $802.11 \mathrm{~g}$ & 2003 & $2.4 \mathrm{GHz}$ & $19 \mathrm{Mpbs}$ & $35 \mathrm{M}$ & $150 \mathrm{M}$ \\
\hline $802.11 \mathrm{~h}$ & 2003 & $5 \mathrm{Ghz}$ & $54 \mathrm{Mpbs}$ & $30 \mathrm{M}$ & $100 \mathrm{M}$ \\
\hline $802.11 \mathrm{n}$ & 2008 & $2.4 \mathrm{GHz}-5 \mathrm{Ghz}$ & $248 \mathrm{Mpbs}$ & $70 \mathrm{M}$ & $250 \mathrm{M}$ \\
\hline $802.11 \mathrm{y}$ & 2008 & $3.7 \mathrm{Ghz}$ & $54 \mathrm{Mpbs}$ & $500 \mathrm{M}$ & $5000 \mathrm{M}$ \\
\hline $802.11 \mathrm{ac}$ & 2013 & $5 \mathrm{Ghz}$ & $433 \mathrm{Mpbs}$ & & \\
\hline
\end{tabular}

As it was seen on the Table1, there is a big increase on information, institution or to be available to process or historical development of Wireless LAN Standards, data disclosure to be provided to property), integrity (removal speed and wireless coverage areas and the opportunity of of the guarantee feature of the accuracy of the existence users to reach the data faster and to benefit from the larger and completeness) and usability ( the feature is not wireless network systems has increased the use of these available at the request of a competent authority) systems. Naturally, several vulnerabilities have appeared protection, the right technology, knowledge by using the in currently heavily used wireless network systems and right purpose and the right way in any medium, preventing security needs began to emerge in the wireless network

\section{SECURITY WIRELESS NETWORKS AND USES METHODS}

the acquisition by undesirable persons, persons and institutions threats they may encounter when using this technology and making the analysis of the hazard is defined as taking the prerequisite measures .

The ISO / IEC 27001 security, the confidentiality of Equivalent Privacy (WEP Wired) information assets of an organization (unauthorized 
when the standard is set as 64-bit encryption using the and causes system vulnerabilities. development process has increased to 128-bit encryption Service Set Identifier( SSID)

and security for up to 256 bits, but has been widely used A service set identifier, is the name of the wireless 128-bit. Despite the development process used by many network broadcast. The name of the broadcast service set security vulnerabilities due to be officially since 2004, it identifier with the feature of wireless network card can be has been proposed.

\section{Wi-Fi-Protected Access (WPA)}

Wi-Fi-Protected Access began to use in 2003 is a Wi-Fi Standard which is developed to completely eliminate the WPA,WEP openings. WPA-PSK(Pre-shared Key) gained a great advantage against WEP by using 256 bit switching technology also monitored the traffic between user and client by adding a key to each transmitted packet with Temporal Key Integrity Protocol. Despite these progresses, since WEP used RC4 Algorithm, in a short time, Security vulnerabilities and Security Weakness have appeared.

\section{Wi-Fi-Protect Access II(WPA2)}

Wi-Fi Protected Access 2 has been used as of 2006. Using the AES algorithm and CCMP (conter cipher mode with block chaining message authentication code protocol) is used instead of TKIP. It is much more powerful encryption method than CCMP TKIP. WPA2 is the most important standard recommended to use but an opening was found in WPA2 and the way to take advantage of this vulnerability, you must be connected to the network.

\section{Media Access Control (MAC)}

MAC (Media Access Control-Media Access Control) address is defined as Unique MAC Address defining itself with letters between A-F and numbers between 0-9 of a hardware device that wants to connect to system in wired and wireless network system. Thanks to MAC Address, MAC Addresses and credentials of users are added to Access Point Controller Device, Access Point. With the Help of The MAC Address Authorization, Uses can access Network System if MAC Address is true or attached in the system. MAC Address Authorization was a very important authorization method in the pastbut nowadays it is generally preferred in non-continuously variable Institution which has low user number. In large organizations updating, controlling and managing MAC Addresses is difficult. In addition, by listening to network, obtaining or changing the MAC Addresses of Involved users in system makes it difficult to identify this process perceived by the client. Once user selected this broadcast, they can get into system with password, involved users can communicate and share the data between the other users and hardware in the same broadcast. Hardware that broadcast SSID broadcasts to a specific area, Different users in the concerned area can see the name of the SSID to reach the system. Therefore SSID hardware chooses the option that disables the name of the broadcast for safety. In this way, security can be provided on a wireless network.

\section{1x Authentication}

802.1x Authentication is a port-based authentication standard to help improve security of wired and wireless networks. 802.1x uses an authentication to verify users and to provide them a network access. In wireless network 802.1x can work with WPA,WPA2 and WEP keys.This type of authentication is typically used when connecting to the work area network[3].

In $802.1 \mathrm{x}$ wireless network system gets included in control of identity by A client requesting connection on Access Point (AP) Extensible Authentication Protocol (EAP) sends a start message, if successful it reaches the system ,if fails it cannot reach the system because the port that it will use to get in is closed[4].

\section{Extensible Authentication Protocol (EAP)}

With EAP a random authentication mechanism authenticates a remote access connection. The exact authentication scheme is determined by the consensus between remote access client or the Remote Authentication Dial-In User Service [RADIUS] server. EAP allows for an open-ended conversation between the remote access client and the authenticator. Interview consists of the information which is requested by the authenticator and responses of the remote client. When all questions have been answered successfully, it passed the remote access client authentication. A specific EAP authentication scheme is termed an EAP type. EAP authentication methods such as MD5, TLS, TTLS, PEAP, LEAP are used [5].

Table-2 Comparison of EAPType

\begin{tabular}{|l|l|l|l|l|l|}
\hline & MD5 & TLS & TTLS & PEAP & LEAP \\
\hline Standard & Open & Open & Open & Open & Company \\
\hline Client Certificate & $\mathrm{x}$ & $\checkmark$ & $\mathrm{X}$ & $\mathrm{x}$ & $\mathrm{x}$ \\
\hline Service Certificate & $\mathrm{x}$ & $\square$ & $\checkmark$ & $\checkmark$ & $\mathrm{x}$ \\
\hline Security & $\mathrm{x}$ & Strong & Strong & Strong & Weak \\
\hline & Open Text & $\begin{array}{l}\text { Active } \\
\text { directory }\end{array}$ & $\begin{array}{l}\text { Token systems, } \\
\text { SQL,LDAP }\end{array}$ & $\begin{array}{l}\text { Active Directory, } \\
\text { NT Domain }\end{array}$ & $\begin{array}{l}\text { Active Directory, } \\
\text { NT Domain }\end{array}$ \\
\hline Dser DATABASE & Password & $\square$ & $\square$ & $\square$ & $\square$ \\
\hline Mutual Authentication & $\mathrm{x}$ & $\mathrm{x}$ & $\square$ & $\square$ & $\square$ \\
\hline
\end{tabular}

As it was seen on the Table-2 comparison of Eap,802.1x standard EAP authentication methods have been examined and the method of EAP-PEAP, and in addition, have been proposed for use with EAP-MSCHAPV2. 


\section{UNIVERSITY WIRELESS SECURITY METHOD}

Wireless Network Security methods were examined, due to the security weakness in used network safety methods, resistance against the attacks which were made, encryption methods and its importance in today's technology, 802.1x was proposed for Universities. In addition to the standard, to be raised to a higher level of wireless network security enhanced by virtual network architecture and design examples for use in university design method has been created and has been implemented in the Fatih Sultan Mehmet Foundation University. Due to faculties, institutes, departments located in the University and professors, administrative staff, number of students, the most appropriate authentication and network security standard IEEE 802.1x was adopted. In addition to this standard by introducing the virtual network architecture because of differences in user, created separate virtual networks for different user groups and virtual networks to communicate between each other restrictions are implemented.

An additional security system was created on the virtual network architecture and the network, packet take-give, bandwidth; broadcast control has become more manageable by making network traffic more flexible. Below, one can see the methods for Universities and basic features and concepts of network which is designed for FSMVU.

\subsection{Virtual LocalArea Network}

Virtual local area network (LAN) is connected to the network on a wired or wireless network system.

\section{University Virtual Local Area Network Design}

Analyses were performed according to security level of user groups in the wireless network architecture and as a result of these analyses the proposed virtual network design was given in the Table-3.

Table-0 VLAN Sample Design Table

\begin{tabular}{|l|l|l|l|l|l|}
\hline VLAN Name & Tag Id & Ip Address & Net mask & Default Gateway & DHCP Server \\
\hline Student_vlan & 2000 & 10.10 .0 .2 & 255.255 .255 .0 & 10.10 .0 .1 & 10.10 .0 .1 \\
\hline Academy_vlan & 2001 & 10.10 .1 .2 & 255.255 .255 .0 & 10.10 .1 .1 & 10.10 .1 .1 \\
\hline Bim_vlan & 2002 & 10.10 .2 .2 & 255.255 .255 .0 & 10.10 .2 .1 & 10.10 .2 .1 \\
\hline Administrative_vlan & 2003 & 10.10 .3 .2 & 255.255 .255 .0 & 10.10 .3 .1 & 10.10 .3 .1 \\
\hline Server_vlan & 2004 & 10.10 .4 .2 & 255.255 .255 .0 & 10.10 .4 .1 & 10.10 .4 .1 \\
\hline
\end{tabular}

\subsection{Lightweight Directory Access Protocol (LDAP)}

Lightweight Directory Access Protocol is a directory service standard. The information contained in the directory access protocol and structure of the database is called nominal. Directory access protocol information in the database is described as a list that contains data about each of the objects and has an ordering logic. Openldap, Sun directory server and directory services such as Microsoft Active Directory can be used for LDAP [7].

\section{Active Directory (A.D)}

The directory service network is a network management system that we can edit permission and authorization of users who can reach any kind of data, shared resources, and hardware devices on the network system. Another important feature of the directory service is the definition of user and group users can access the directory service after a certain authentication. Different policies can be taken for users and groups according to the authentication.

Table-4 The Directory Service (AD) University Design Sample

\begin{tabular}{|l|l|l|}
\hline Organization Unit & Groups & Users \\
\hline Administrative & Information Technology .D & ITD.User1 \\
& Student Affairs .D & SAD.User1 \\
\hline Academic staff & Faculty of Engineering & FE.User1 \\
& Institu of Fine Arts & IFA.User1 \\
\hline Student & Faculty of Arts & FA.stuUser1 \\
& Vocational School & VS.stuUser1 \\
\hline Server & & S.User1 \\
\hline Guest & & GUser1 \\
\hline
\end{tabular}

The directory service design model for universities are given in the Table-4

\section{The directory service certification service}

Directory certificate service is a service used in software security systems and makes use of public key technology with public key infrastructure (PKI Public Key Infrastructure).

\subsection{Authentication and Authorization server (RADIUS Server)}

RADIUS (Remote Authentication Dial-in User Service) makes the process for users who connect remotely server user name-password authentication reporting/ access time accounting and authorization 
Radius can be used as server for free radius and like Microsoft network policy server[8].

\section{Network Policy Server (NPS)}

In the draft for University, Microsoft NPS service was used for RADIUS because of simplicity of management, high level of security and the ability to configure it in a few easy steps. By NPS, Network access policies were created in the entire university for authorization procedures, connect request authorization, client health. For wireless connections EAP methods -as we can see the data in the Table- 2 in $802.1 \mathrm{x}$ standard can be enlarged protectively due to the high level of safety. Protected Extensible Authentication Protocol (PEAP), and in addition to PEAP for secure password authentication is used with MS-CHAP v2 together.

\subsection{Access Point Controller}

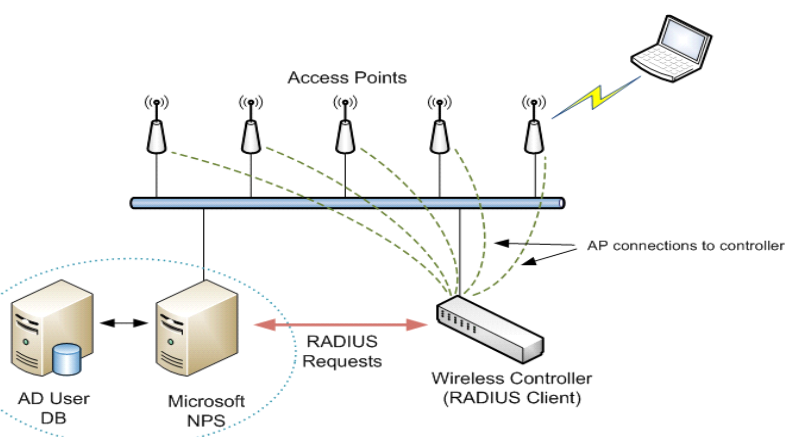

Figure-1 Access Point Controller

In Figure-1 Communication between the access point and radius server is managed by the access point controller. Access Point (AP) is a hardware device in the wireless network system that SSID was broadcasted and connection re-question of users over the broadcasted SSID. AP is a device that controls, reports and manages all AP Devices and when connection request was transmitted to AP devices, it tells us by which configuration $802.1 \mathrm{x}$ will be affected. The access point controller designed on virtual networks, it is necessary configurations to communicate with the Network Policy Server.

\subsection{Switch}

It constitutes an important part of the network switch and routing data communications in a wireless network system. Even if the wireless network broadcast was made on the air, we must create the configuration on switch key. Because devices such as AP devices, access point, firewall and network policy server communicate via Ethernet port or Fiber connection.

\subsection{Firewall}

The firewall is a hardware and software system that is developed to protect the resources on the network system against the attacks on the internal network system or another network system. Generally it can be inferred as network solution that controls traffic between the external and internal network according to the rules of institution. Thus, On that draft , it needs to be defined that on which virtual network number it will connect the internet by creating zones and Interfaces and also IP address needs to be defined by forming DHCP to identify the traffic on the virtual networks and for user to get an IP address according to the rules we made

\section{TESTING THE STRUCTURE OF THE DESIGNED NETWORK}

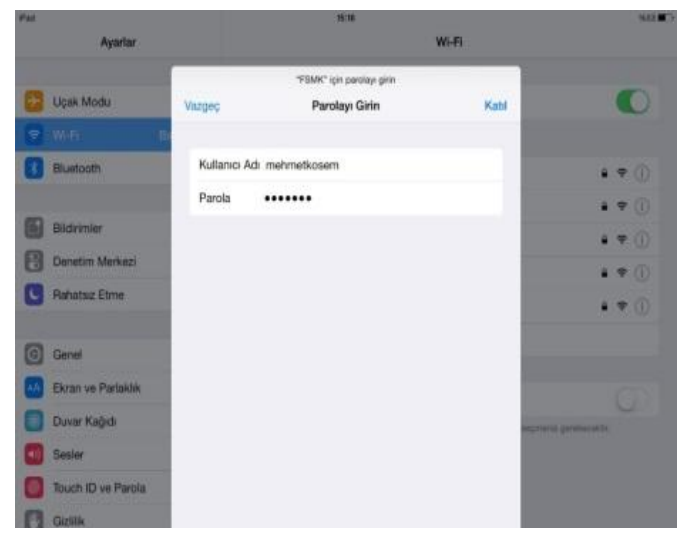

Figure-2 Username and Password Screen
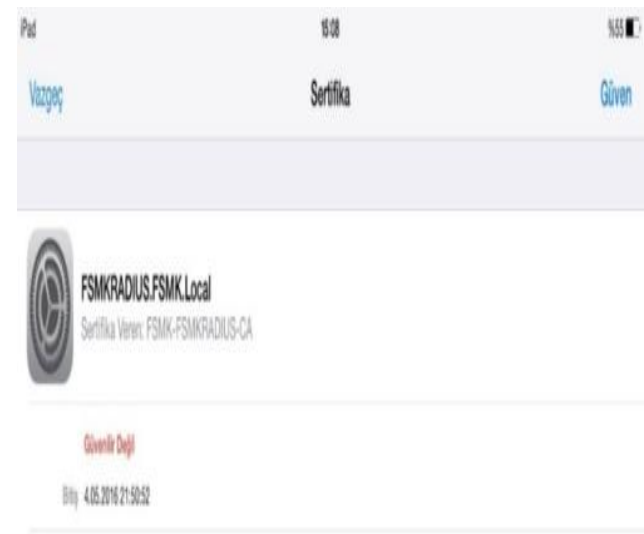

Desaredahint

Figure-3 Certification

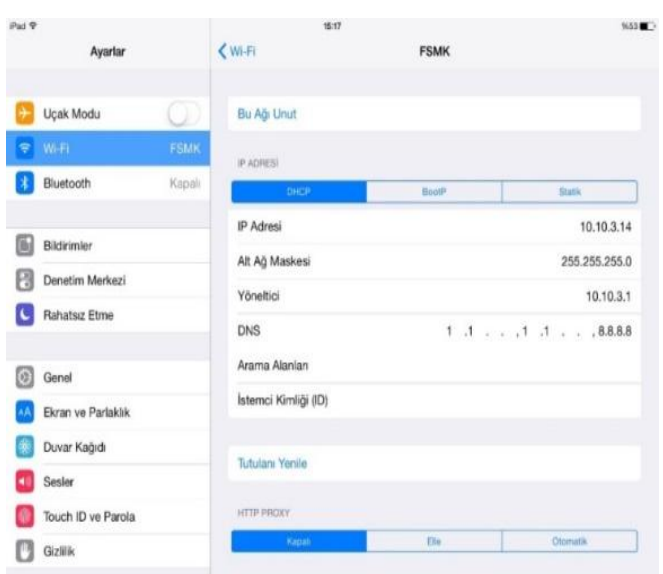

Figure-0 Wireless Network Information

In Figure-4, after entry and certification (Figure-2 and Figure-4), user Mehmet Kosem, in the 802.1x standard test, His identity was approved Firewall transferred the user according to virtual network number of user to administrative _test virtual network and assigned the user 
IP Address as 10.10.3.14,default gateway 10.10.3.1and User could reach IOS operating system.

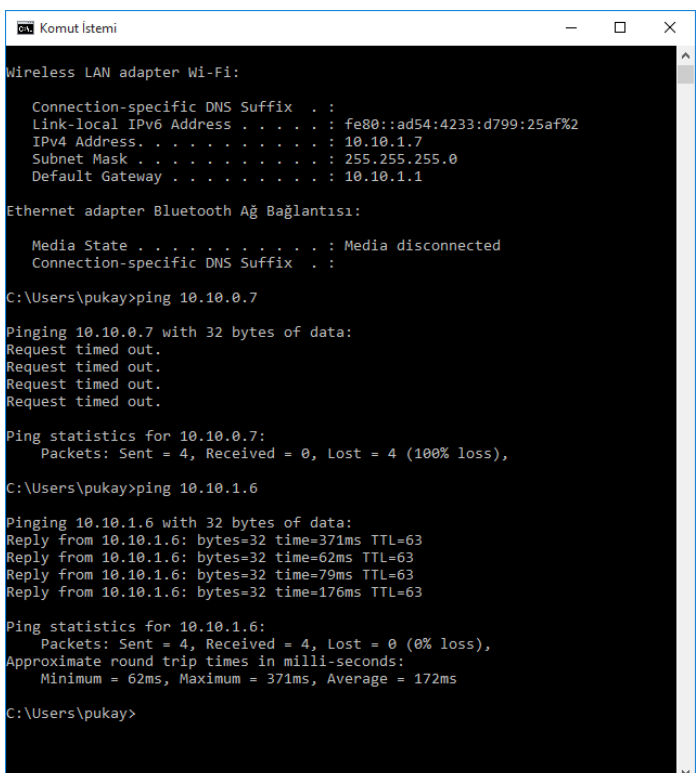

Figure-5 IP Address Configuration and Ping Information

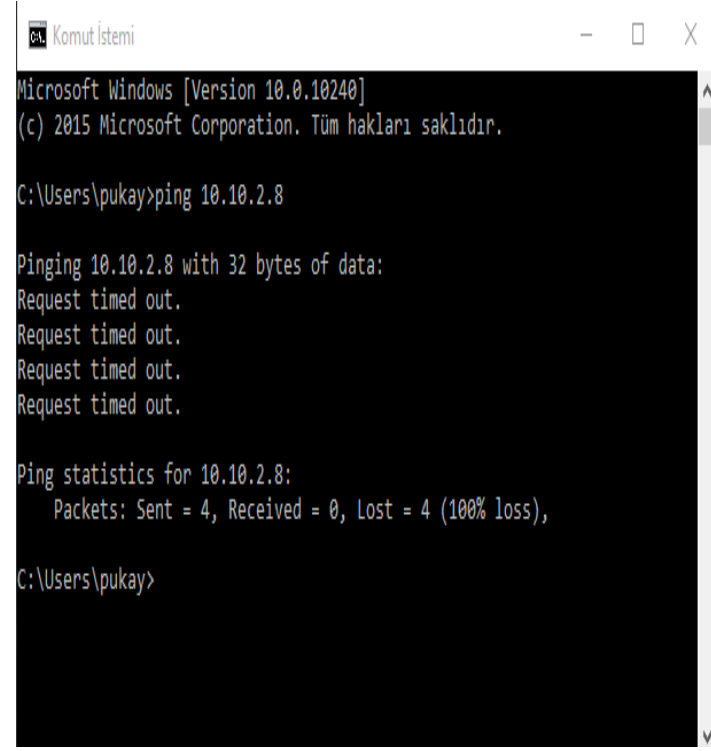

Figure-6 Ping Information

After his identity was approved in $802.1 \mathrm{x}$ standard, the user Yocal reached the system on academic virtual network and he received the 10.10.1.7. But he couldn't send data to (As seen in Figure-5) 10.10.0.7 and (As seen in Figure-6)10.10.2.8 IP addresses. Although he could communicate with the academic network since he was in the network, he couldn't communicate with the Student and Administrative Networks. Thus Communication security between virtual networks was increased

\section{CONCLUSIONS AND RECOMMENDATIONS}

The use of a wireless network with the development of technology is increasing every day. With the increase in the utilization rate of authentication and many studies are performed on wireless network security. In this study, wireless network security methods have been worked out and the $802.1 \mathrm{x}$ standard for wireless network authentication and access control systems have been proposed. In this study, as a result of the applications of 802.1x standard in University wireless network Security, Designs that has hardware and software-free material have been proposed and It is claimed that use of $802.1 \mathrm{x}$ standard after identity approval on wireless network must be together with virtual network to increase the security of Network System. Also 802.1x standards was examined with Eap (PEAP) and since still cannot be revealed the results of encryption and listening to the data when used with EAP MSCHAPV2, using them together has been suggested.

In this study, wireless network security authentication server, certificate server, directory service server, Access Point controller, network switching equipment, test environment of firewall devices have been created and the configuration process for $802.1 \mathrm{x}$ standards at the university were carried out. After the test, results have been reported and methods have been proposed for the university.

\section{REFERENCES}

1. ERKINAY M,Wireless Networks and Wireless Network Security, YuzuncuYilUniversity,Unpublished Master Thesis, Istanbul, 2006,

2. CALDER, A., "Nine Steps to Success: An ISO 27001 Implementation Overview", IT GovernenceInstitute Conference, (2006)

3. http://windows.microsoft.com/tr-tr/windows/what-are-wirelessnetwork-security-methods\#1TC=windows-7

4. tr.wikipedia.org/wiki/IEEE_802.1X

5. http://csirt.ulakbim.gov.tr/dokumanlar/Ag_Kimlik_Denetimi.pdf

6. http://Ulakbim.gov.tr

7. http://docs.comu.edu.tr/howto/ldap-howto-intro.html

8. yunus.hacettepe.edu.tr/ b0145561/bilg_aglar.html

9. http://www.chip.com.tr/haber/merakli-komsulara-son-wi-fikorumali-erisim_41190_2.html 